\title{
No Be binary component in the eclipsing binary DW Carinae (Research Note)
}

\author{
P. Harmanec \\ Astronomical Institute of the Charles University, Faculty of Mathematics and Physics, V Holešovičkách 2, 18000 Praha 8 , \\ Czech Republic \\ e-mail: hec@sirrah.troja.mff.cuni.cz
}

Received 8 November 2007 / Accepted 22 December 2007

\section{ABSTRACT}

\begin{abstract}
In a recent excellent study by Southworth and Clausen, refined orbital elements and basic physical properties of DW Carinae were derived. A suggestion was made that this binary is a Be-star system since a double, $\mathrm{H} \alpha$ emission line was observed. I argue that the observed $\mathrm{H} \alpha$ emission is nebular emission from the Carina nebula and that neither of the binary components of DW Carinae is a Be star.
\end{abstract}

Key words. binaries: eclipsing - stars: early-type - stars: fundamental parameters - stars: emission-line, $\mathrm{Be}-$ stars: individual: DW Carinae

\section{Introduction}

DW Carinae (HDE 305543, CD-59³251) is a detached eclipsing and a double-lined binary and a member of the open cluster Collinder 228. It consists of two similar early-B stars orbiting each other with a period of 1.32775 . Southworth \& Clausen (2007) published an excellent study of DW Carinae (DW Car). They showed that careful application of disentangling provides a superior determination of orbital elements, if results are compared with alternative methods.

\section{The origin of the $\mathrm{H} \alpha$ emission}

Southworth \& Clausen (2007) called DW Carinae the Be star system because of double, $\mathrm{H} \alpha$ emission line was detected at a level of forty per cent above the measured continuum. A puzzling fact about their observations is that neither the double emission line, nor the central absorption line, indicate any change in radial velocity $(R V)$, and that the RV is equal to the systemic velocity of DW Car.

It is well-established that in most Be stars that are members of binary systems, the RV measured on the wings of the (usually) double $\mathrm{H} \alpha$ emission line follows the orbital motion of the Be component. This is the case of $\kappa$ Dra (HD 109387) (Juza et al. 1991), $\varphi$ Per (HD 10516) (Božić et al. 1995), V839 Her (4 Her, HD 142926) (Koubský et al. 1997), $\gamma$ Cas (HD 5394) (Harmanec et al. 2000; Miroshnichenko et al. 2002), $\delta$ Sco (HD 143275) (Miroshnichenko et al. 2001), V832 Cyg (59 Cyg, HD 200120) (Harmanec et al. 2002), or $\pi$ Aqr (HD 212571) (Bjorkman et al. 2002) among others. Božić et al. (1995) and Harmanec (2003) both argue that the RV measured using the steep $\mathrm{H} \alpha$ emissionline wings represents the most accurate measurement of the true Be-star orbital motion.

In some more complicated cases such as $\beta$ Lyr (HD 174638), the bulk of the emission is associated with bipolar jets (Harmanec et al. 1996). However, even in such cases, the radial velocity of the $\mathrm{H} \alpha$ emission exhibits phase-locked changes.
I note that the light curves of binaries, which contain a $\mathrm{Be}$ star, usually exhibit secular variations. In contrast, the light curve of DW Carinae stable, without any obvious peculiarities.

Southworth \& Clausen (2007) suggest that the observed emission of DW Carinae originates from a circumbinary disk located in the orbital plane. I argue that this is also improbable. As discussed quantitatively by Thackeray (1971) and Kř́iž \& Harmanec (1975), a projection of such a circumstellar disk onto one of the binary components would result in RV variations of the central absorption in phase with the orbital radial-velocity curve but with strongly reduced amplitude. Such a behaviour is observed for V367 Cyg (HD 198287) (Heiser 1961) and FY Vel (HD 72754) (Thackeray 1971).

I estimate the observed RV of such an envelope around DW Car, following the methology of Kř́ž \& Harmanec (1975). I assume that the envelope around the DW Carinae system can be modelled as a cylindrical envelope revolving at an effective distance $d$ about the common centre of gravity of the binary. If the binary components have masses $M_{1}$ and $M_{2}$, the Keplerian rotation of the envelope at larger distances $d$ can be approximated as

$v_{\mathrm{env}}=\left(\frac{G\left(M_{1}+M_{2}\right)}{d}\right)^{\frac{1}{2}}$.

Kř́ž \& Harmanec (1975) have shown that the RV of the absorption line arising from the projection of a part of the envelope against the disk of the brighter component 1 will be given by

$\frac{R V_{\text {env. }}}{R V_{1}}=\left(\frac{a^{3}}{d^{3}}\right)$,

where $a$ is the binary separation. Measuring the RVs of the observed $\mathrm{H} \alpha$ line profiles in the enlarged plot of Fig. 1 of Southworth \& Clausen (2007), I find that RVs of the $V$ emission peak, central absorption and the $R$ emission peak, respectively, are $-32 \mathrm{~km} \mathrm{~s}^{-1},-9 \mathrm{~km} \mathrm{~s}^{-1}$, and $+14 \mathrm{~km} \mathrm{~s}^{-1}$. I assume that the peak separation gives a fair estimate of the rotational velocity 
of the disk. Assuming the above values for DW Car, and the inclination $i=85.72$, this corresponds to a rotational velocity of $v_{\text {env. }}=23 \mathrm{~km} \mathrm{~s}^{-1}$. Using Eq. (1) and the binary masses derived by Southworth $\&$ Clausen (2007) I obtain $d=7875 R_{\odot}$. From Eq. (2) it then follows that $R V_{\text {env }}=1.5 \times 10^{-6} \mathrm{~km} \mathrm{~s}^{-1}$. This is in agreement with the constant RV measured for the $\mathrm{H} \alpha$ emission line. However, the estimated effective radius of the putative circumstellar disk is much larger than the typical disk radii derived for known Be stars, using a variety of methods. These radii are usually smaller than $100 R_{\odot}$ (Rinehart et al. 1999; Waters et al. 1987; Gehrz et al. 1974). It is clear that for the above-estimated very large effective distance from the binary, such a circumbinary structure would become a part of the Carina nebula and its Balmer emission would probably be excited by the summed radiation of a number of the cluster stars.

A more convincing and natural explanation for the $\mathrm{H} \alpha$ emission of DW Carinae observed by Southworth \& Clausen (2007) is that it is nebular emission from the Carina nebula. Double, nebular $\mathrm{H} \gamma$ emission lines from the Carina nebula were reported in long-exposure coudé spectrograms of the O5V star HD 93204 (CD-59 3293 ) by Walborn \& Hesser (1975) with radial velocites of -30 to $-35 \mathrm{~km} \mathrm{~s}^{-1}$ and +2 to $+6 \mathrm{~km} \mathrm{~s}^{-1}$. I note the similarity of the observed $\mathrm{H} \alpha$ emission profile of DW Carinae and the $\mathrm{H} \gamma$ emission profile of HD 93204 as well as similarity of my $\mathrm{H} \alpha$ emission-peak RV measurements of DW Carinae to those for the $\mathrm{H} \gamma$ emission of HD 93204. As Walborn \& Hesser (1975) point out, the Carina nebula is a very complex structure illuminated by a number of very hot stars.

I also note that the formation of a circumstellar envelope surrounding DW Carinae appears improbable in the light of the accurate results of Southworth \& Clausen (2007): they found that data for both components is well-described by the isochrones of stellar-evolutionary models for an age of $6 \mathrm{Myr}$ which is about one third of the main-sequence lifetime of $11 M_{\odot}$ stars. The estimated radii of the Roche lobes surrounding both components are about 5.4 and $5.2 R_{\odot}$, significantly larger than the observed stellar radii. Therefore, no large-scale mass exchange has yet occurred in the system. The stellar wind of stars in this mass range is not particularly strong and even if it were, it is not obvious how an envelope would be formed from a supersonic wind reaching escape velocities. There is also no evidence of variability in the orbital period which would be indicative of a rapid, dynamical evolution of the system.

I conclude that any discussion of the $\mathrm{H} \alpha$ emission observed in the DW Carinae spectra in relation to the (yet unexplained) Be phenomenon should be abandoned. I propose instead that DW Carinae is a normal main-sequence detached binary, and should not be included in any lists of known Be stars.

Acknowledgements. This research was supported by the grant 205/06/0304 of the Czech Science Foundation and the Research Program MSM0021620860 of the Ministry of Education, Youth and Sports of the Czech Republic. The critical remarks of an anonymous referee helped me to sharpen the arguments in favour of the nebular origin of the observed Balmer emission and are gratefully acknowledged.

\section{References}

Bjorkman, K. S., Miroshnichenko, A. S., McDavid, D., \& Pogrosheva, T. M. 2002, ApJ, 573, 812

Božić, H., Harmanec, P., Horn, J., et al. 1995, A\&A, 304, 235

Gehrz, R. D., Hackwell, J. A., \& Jones, T. W. 1974, ApJ, 191, 675

Harmanec, P. 2003, in Publ. Canakkale Onsekiz Mart Univ. New Directions for Close Binary Studies: The Royal Road to the Stars, 3, 221

Harmanec, P., Morand, F., Bonneau, D., et al. 1996, A\&A, 312, 879

Harmanec, P., Habuda, P., Štefl, S., et al. 2000, A\&A, 364, L85

Harmanec, P., Božić, H., Percy, J. R., et al. 2002, A\&A, 387, 580

Heiser, A. M. 1961, ApJ, 134, 568

Juza, K., Harmanec, P., Hill, G. M., Tarasov, A. E., \& Matthews, J. M. 1991, Bulletin of the Astronomical Institutes of Czechoslovakia, 42, 39

Koubský, P., Harmanec, P., Kubát, J., et al. 1997, A\&A, 328, 551

Kříž, S., \& Harmanec, P. 1975, BAICz, 26, 65

Miroshnichenko, A. S., Fabregat, J., Bjorkman, K. S., et al. 2001, A\&A, 377, 485

Miroshnichenko, A. S., Bjorkman, K. S., \& Krugov, V. D. 2002, PASP, 114, 1226

Rinehart, S. A., Houck, J. R., \& Smith, J. D. 1999, AJ, 118, 2974

Southworth, J., \& Clausen, J. 2007, A\&A, 461, 1077

Thackeray, A. D. 1971, MNRAS, 154, 103

Walborn, N. R., \& Hesser, J. E. 1975, ApJ, 199, 535

Waters, L. B. F. M., Cote, J., \& Lamers, H. J. G. L. M. 1987, A\&A, 185, 206 